\title{
Heavy-light quarks interactions in QCD vacuum
}

\section{Mirzayusuf Musakhanov*}

National University of Uzbekistan

E-mail: musakhanov@gmail.com

QCD vacuum instantons induce very strong interactions between light quarks, which generate large dynamical light quark mass $\mathrm{M}$ for initially almost massless quarks and can bound these quarks to produce almost massless pions in accordance with the spontaneous breaking of chiral symmetry $(S \chi \mathrm{SB})$. On the other hand, the QCD vacuum instantons generate heavy-light quark interactions terms, which are responsible for the effects of $S \chi \mathrm{SB}$ in a heavy-light quark system. Summing the re-scattering series that lead to the total light quark propagator and making few further steps, we get the fermionized representation of low-frequencies light quark determinant in the presence of the quark sources, which is relevant for our problems. The next important step in the line of this strategy is to derive the equation and calculate the heavy quark propagator in the instanton media and in the presence of light quarks. This one provide finally the heavy and $N_{f}$ light quark interaction term. As an example, we derive heavy-light quark meson interaction term for the $N_{f}=2$ case. If we take the average instanton size $\rho=0.35 \mathrm{fm}$, and average inter-instanton distance $\mathrm{R}=0.856 \mathrm{fm}$ from our previous estimates, we obtain at LO on $1 / N_{c}$ expansion dynamical light quark mass $\mathrm{M}=570 \mathrm{MeV}$ and instanton media contribution to heavy quark mass $\Delta M=148$ $\mathrm{MeV}$. These factors define the coupling between heavy and light quarks and, certainly, between heavy quarks and light mesons. We will apply this approach to heavy quark and heavy-light quark systems.

XXII International Baldin Seminar on High Energy Physics Problems

15-20 September, 2014

JINR, Dubna, Russia

${ }^{*}$ Speaker. 


\section{Introduction}

One of the most prominent advances of the QCD instanton vacuum model is the correct description of the spontaneous breaking of chiral symmetry $(S \chi \mathrm{SB})$, which is responsible for properties of most light hadrons and nuclei. Instantons induce very strong interactions between light quarks, which generate the dynamical quark mass $\sim 400 \mathrm{MeV}$ for initially almost massless quarks and can bound these quarks to produce almost massless pions in accordance with $S \chi \mathrm{SB}$. In the instanton picture $S \chi \mathrm{SB}$ is due to the delocalization of single-instanton quark zero modes in the instanton medium.

The instanton vacuum field is assumed as a superposition of $N_{+}$instantons and $N_{-}$antiinstantons:

$$
A_{\mu}(x)=\sum_{+}^{N_{+}} A_{\mu}^{I}\left(\zeta_{+}, x\right)+\sum_{-}^{N_{-}} A_{\mu}^{A}\left(\zeta_{-}, x\right)
$$

Here, $\zeta=(\rho, z, U)$ denote respectively (anti)instanton collective coordinates- size, position and color orientation (see reviews $[1,2]$ ). One of the advantages of the instanton vacuum model is that it is characterized by only two parameters: the average instanton size $\rho$ and the average interinstanton distance $R$. The estimates of these quantities are

$$
\begin{aligned}
& \rho \simeq 0.33 \mathrm{fm}, R \simeq 1 \mathrm{fm},(\text { phenomenological) }[1,2], \\
& \rho \simeq 0.35 \mathrm{fm}, R \simeq 0.95 \mathrm{fm},(\text { variational) }[1], \\
& \rho \simeq 0.36 \mathrm{fm}, R \simeq 0.89 \mathrm{fm}, \text { (lattice) }[3] .
\end{aligned}
$$

Our estimates [6] (in the instanton vacuum model with $1 / N_{c}$ corrections taken into account) from the pion decay constant $F_{\pi, m=0}=88 \mathrm{MeV}$ and the quark condensate $\langle\bar{q} q\rangle_{m=0}=-(255 \mathrm{MeV})^{3}$ in the chiral limit [5] are given as:

$$
R \approx 0.856 \mathrm{fm}, \rho \approx 0.35 \mathrm{fm} .
$$

A recent computer simulation [4] of a current mass dependence of QCD observables within the instanton liquid model show that the best correspondence to the lattice QCD data is obtained as $R \approx 0.76 \mathrm{fm}, \rho \approx 0.32 \mathrm{fm}$. Thus within $10-15 \%$ uncertainty, different approaches give similar estimates. In the following we will use our values for $R, \rho$ (1.3).

While the instantons are absolutely important for the light quark physics, for the heavy quarks even the charmed quark mass $m_{c} \sim 1.5 \mathrm{GeV}$ is essentially larger than the typical parameters of the instanton media-the inverse instanton size $\rho^{-1} \approx 600 \mathrm{MeV}$ and the inter-instanton distance $R^{-1} \approx$ $200 \mathrm{MeV}$ and thus the quark mass mainly determines the dynamics of the heavy quarks $[7,8]$. On the other hand, the QCD vacuum instantons generate heavy-light quark interactions terms which are responsible for the effects of $S \chi \mathrm{SB}$ in a heavy-light quark system. Our aim is to derive these interaction terms. First, we have to take into account light quarks effects in the partition function.

\section{Light quark determinant with the quark sources term}

We start from the splitting of the total quark determinant to the low and high frequencies parts as Det $=\operatorname{Det}_{\text {high }} \cdot$ Det $_{\text {low }}$, where Det $t_{\text {high }}$ gets a contribution from fermion modes with Dirac 
eigenvalues from the interval $M_{1}$ to the Pauli-Villars mass $M$, and Det low $_{\text {low }}$ is accounted eigenvalues less than $M_{1}$. The product of these determinants is independent of the scale $M_{1}$. However, we may calculate both of them only approximately. There is a week dependence of the product on $M_{1}$ in the wide range of $M_{1}$, which serves as a check of the approximations [9].

The high-momentum part Det high $_{\text {can }}$ be written as a product of the determinants in the field of individual instantons, while the low-momentum one Det low has to be treated approximately, would-be zero modes being taken into account only.

The next step is to compute the light quark propagator in the instanton media. Our main assumption is the interpolation formula $[6,11]$ :

$$
S_{i}=S_{0}+S_{0} \hat{p} \frac{\left|\Phi_{0 i}><\Phi_{0 i}\right|}{c_{i}} \hat{p} S_{0}, S_{0}=\frac{1}{\hat{p}+i m}, c_{i}=i m<\Phi_{0 i}\left|\hat{p} S_{0}\right| \Phi_{0 i}>.
$$

The advantage of this interpolation is shown by the projection of $S_{i}$ to the zero-modes:

$$
S_{i}\left|\Phi_{0 i}>=\frac{1}{i m}\right| \Phi_{0 i}>,<\Phi_{0 i}\left|S_{i}=<\Phi_{0 i}\right| \frac{1}{i m}
$$

as it must be, while the similar projection of $S_{i}$ given by Ref. [9] has a wrong component, negligible only in the $m \rightarrow 0$ limit.

Summing the re-scattering series that lead to the total quark propagator and making few further steps, we get the fermionized representation of low-frequencies light quark determinant in the presence of the quark sources, which is relevant for our problems, in the form $[6,11]$ :

$$
\begin{aligned}
& \operatorname{Det}_{\text {low }} \exp \left(-\eta^{+} S \eta\right)= \\
& \int \prod_{f} D \psi_{f} D \psi_{f}^{\dagger} \exp \int \sum_{f}\left(\psi_{f}^{\dagger}\left(\hat{p}+i m_{f}\right) \psi_{f}+\psi_{f}^{\dagger} \eta_{f}+\eta_{f}^{+} \psi_{f}\right) \prod_{f} \prod_{ \pm}^{N_{ \pm}} V_{ \pm, f}\left[\psi^{\dagger}, \psi\right],
\end{aligned}
$$

where

$$
V_{ \pm, f}\left[\psi^{\dagger}, \psi\right]=i \int d^{4} x\left(\psi_{f}^{\dagger}(x) \hat{p} \Phi_{ \pm, 0}\left(x ; \zeta_{ \pm}\right)\right) \int d^{4} y\left(\Phi_{ \pm, 0}^{\dagger}\left(y ; \zeta_{ \pm}\right)\left(\hat{p} \psi_{f}(y)\right)\right.
$$

The averaging over collective coordinates $\zeta_{i, \pm}$ of $\operatorname{Det}_{\text {low }} \exp \left(-\eta^{+} S \eta\right)$ is a rather simple procedure, since the low density of the instanton medium $\left(\pi^{2}\left(\frac{\rho}{R}\right)^{4} \sim 0.1\right)$ allows us to average over positions and orientations of the instantons independently. This one leads to the light quark partition function $Z\left[\eta, \eta^{+}\right]$. From (2.3) at $N_{f}=1$ and $N_{ \pm}=N / 2$ it is exactly given by

$$
\begin{aligned}
& Z\left[\eta, \eta^{+}\right]=e^{-\eta^{+}(\hat{p}+i(m+M(p)))^{-1} \eta} \exp \left[\operatorname{Tr} \ln (\hat{p}+i(m+M(p)))+N \ln \frac{N / 2}{\lambda}-N\right], \\
& N=\operatorname{Tr} \frac{i M(p)}{\hat{p}+i(m+M(p))}, M(p)=\frac{\lambda}{N_{c}}(2 \pi \rho F(p))^{2} .
\end{aligned}
$$

Here the form-factor

$$
F(p)=2 z\left(I_{0}(z) K_{1}(z)-I_{1}(z) K_{0}(z)-\frac{1}{z} I_{1}(z) K_{1}(z)\right)
$$

$\left(I_{0}, I_{1}, K_{0}, K_{1}\right.$ are the modified Bessel functions, $\left.z=p \rho / 2\right)$ is given by Fourier-transform of the zero-mode. The coupling $\lambda$ and the dynamical quark mass $M(p)$ are defined by Eq. (2.6).

At $N_{f}>1$ and in the saddle-point approximation (no meson loops contribution) $Z\left[\eta_{f}, \eta_{f}^{+}\right]$has a similar form to Eq. (2.5). 


\section{Heavy quark propagator}

The next important step in the line of this strategy is to calculate the heavy quark propagator in the instanton media and in the presence of light quarks. We will extend the equation for the heavy quark propagator in the instanton media previously derived in Refs. [7, 10].

The heavy quark Lagrangian in the external gluon field and in Euclid space is given by $L_{H}=\Psi^{+}\left(\hat{P}+i m_{H}\right) \Psi, P=p-g A$. We make a Foldy-Wouthuysen transformation accordingly[8]: $\Psi(x)=\exp \left(-m_{H} \gamma_{4} x_{4}+O\left(1 / m_{H}\right)\right) Q(x)$, which leads to

$$
L_{H}=\Psi^{+}\left(\hat{P}+i m_{H}\right) \Psi=Q^{+} \gamma_{4} P_{4} Q+Q^{+} Q_{1} Q, Q_{1}=\frac{\vec{P}^{2}}{2 m_{H}}-\frac{\vec{\sigma} \vec{B}}{2 m_{H}}, \vec{B}=\operatorname{rot} \vec{A} .
$$

In the present case we neglect $O\left(1 / m_{H}\right)$ terms and define the heavy quark propagator as:

$$
\begin{aligned}
& S_{H}=\frac{1}{Z} \int \prod_{f} D \psi_{f} D \psi_{f}^{\dagger} \exp \int \sum_{f}\left(\psi_{f}^{\dagger}\left(\hat{p}+i m_{f}\right) \psi_{f}\right) \prod_{ \pm}^{N_{ \pm}}<\prod_{f} V_{ \pm, f}\left[\psi^{\dagger}, \psi\right]>w\left[\psi, \psi^{\dagger}\right], \\
& <\prod_{f} V_{ \pm, f}\left[\psi^{\dagger}, \psi\right]>\equiv \int d \zeta_{ \pm} \prod_{f} V_{ \pm, f}\left[\psi^{\dagger}, \psi\right] \\
& w\left[\psi, \psi^{\dagger}\right]=\left\{\prod_{ \pm}^{N_{ \pm}}<\prod_{f} V_{ \pm, f}\left[\psi^{\dagger}, \psi\right]>\right\}^{-1} \int \prod_{ \pm}^{N_{ \pm}} d \zeta_{ \pm}\left\{\prod_{ \pm}^{N_{ \pm}} V_{ \pm, f}\left[\psi^{\dagger}, \psi\right]\right\} \frac{1}{\theta^{-1}-\sum_{i} a_{i}} \\
& <t|\theta| t^{\prime}>=\theta\left(t-t^{\prime}\right),<t\left|\theta^{-1}\right| t^{\prime}>=-\frac{d}{d t} \delta\left(t-t^{\prime}\right), a_{i}(t)=i A_{i, \mu}(x(t)) \frac{d}{d t} x_{\mu}(t) .
\end{aligned}
$$

Accordingly, we derive the inverse of $w\left[\psi^{\dagger}, \psi\right]$ as [7]

$$
\begin{aligned}
& w^{-1}\left[\psi, \psi^{\dagger}\right]=\theta^{-1}+\frac{N}{2} \sum_{ \pm} \frac{1}{<\prod_{f} V_{ \pm, f}\left[\psi^{\dagger}, \psi\right]>} \int d \zeta_{ \pm} \prod_{f} V_{ \pm, f}\left[\psi^{\dagger}, \psi\right]\left(\theta-a_{ \pm}^{-1}\right)^{-1}+O\left(N^{2} / V^{2}\right) \\
& =\theta^{-1}-\frac{N}{2} \sum_{ \pm} \frac{1}{<\prod_{f} V_{ \pm, f}\left[\psi^{\dagger}, \psi\right]>} \Delta_{H, \pm}\left[\psi^{\dagger}, \psi\right]+O\left(N^{2} / V^{2}\right),
\end{aligned}
$$

where

$$
\Delta_{H, \pm}\left[\psi^{\dagger}, \psi\right]=\int d \zeta_{ \pm} \prod_{f} V_{ \pm, f}\left[\psi^{\dagger}, \psi\right] \theta^{-1}\left(w_{ \pm}-\theta\right) \theta^{-1}
$$

represent the interactions of heavy and $N_{f}$ light quarks and $w_{ \pm}=\frac{1}{\theta^{-1}-a_{ \pm}}$- heavy quark propagator in the single (anti)instanton field. Finally we get the quark propagator in the instanton media with account of light quarks as

$$
S_{H}=\frac{1}{\theta^{-1}-\lambda \sum_{ \pm} \Delta_{H, \pm}\left[\frac{\delta}{\delta \eta}, \frac{\delta}{\delta \eta^{+}}\right]} \exp \left[-\eta^{+}(\hat{p}+i(m+M(p)))^{-1} \eta\right]_{\left.\right|_{\eta=\eta^{+}=0}} .
$$

We approximate Eq.(3.5), neglecting by overlapping diagrams as

$$
\begin{aligned}
& S_{H}^{-1} \approx \theta^{-1}-\lambda \sum_{ \pm} \Delta_{H, \pm}\left[\frac{\delta}{\delta \eta}, \frac{\delta}{\delta \eta^{+}}\right] \exp \left[\eta^{+}(\hat{p}+i(m+M(p)))^{-1} \eta\right]_{\left.\right|_{\eta=\eta}+=0} \\
& =\theta^{-1}-i \operatorname{tr} \int \frac{d^{4} k_{1}}{(2 \pi)^{4}} \frac{\lambda(2 \pi \rho)^{2} F^{2}\left(k_{1}\right)}{N_{c}\left(\hat{k}_{1}+i\left(m+M\left(k_{1}\right)\right)\right)} \frac{1}{2 N_{c}} \sum_{ \pm} \int d^{4} z_{ \pm} \operatorname{tr}_{c}\left(\theta^{-1}\left(w_{ \pm}-\theta\right) \theta^{-1}\right) \\
& =\theta^{-1}-\frac{N}{2 V N_{c}} \sum_{ \pm} \int d^{4} z_{ \pm} \operatorname{tr}_{c}\left(\theta^{-1}\left(w_{ \pm}-\theta\right) \theta^{-1}\right) .
\end{aligned}
$$


Then, in this approximation Eq. (3.7) exactly coincides with the similar one from [7].

\section{Light-heavy quarks interaction term}

Now rewrite Eq. (3.5) by introducing heavy quark fields $Q, Q^{\dagger}$ :

$$
\begin{aligned}
& S_{H}=e^{[-\operatorname{Tr} \ln (\hat{p}+i(m+M(p)))]} \int D \psi D \psi^{\dagger} D Q D Q^{\dagger} Q Q^{\dagger} \exp \left[\left(\psi^{\dagger}(\hat{p}+i(m+M(p))) \psi\right)\right. \\
& \left.+Q^{\dagger}\left(\theta^{-1}-\lambda \sum_{ \pm} \Delta_{H, \pm}\left[\psi^{\dagger}, \psi\right]\right) Q-\operatorname{Tr} \ln \left(\theta^{-1}-\lambda \sum_{ \pm} \Delta_{H, \pm}\left[\psi^{\dagger}, \psi\right]\right)\right]
\end{aligned}
$$

where the third term represent the (negligible) contribution of the heavy quark loops, while the second one is the heavy and light quarks interaction action.

The heavy and $N_{f}$ light quark interaction term explicitly is given by the expression:

$$
\begin{aligned}
& S_{Q q}=-\lambda \sum_{ \pm} Q^{\dagger} \Delta_{H, \pm}\left[\psi^{\dagger}, \psi\right] Q=-i \lambda \sum_{ \pm} \int d^{4} z_{ \pm} d U_{ \pm} \prod_{f=1}^{N_{f}} \frac{d^{4} k_{f}}{(2 \pi)^{4}} \frac{d^{4} q_{f}}{(2 \pi)^{4}} \exp \left(i\left(q_{f}-k_{f}\right) z_{ \pm}\right) \\
& \times \frac{(2 \pi \rho)^{2} F\left(k_{f}\right) F\left(q_{f}\right)}{8} \psi_{f, a_{f} \alpha_{f}}^{+}\left(k_{f}\right)\left(\gamma_{\mu_{f}} \gamma_{v_{f}} \frac{1 \pm \gamma_{5}}{2}\right)_{\alpha_{f} \beta_{f}}\left(U_{ \pm, i_{f}}^{a_{f}}\left(\tau_{\mu_{f}}^{\mp} \tau_{v_{f}}^{ \pm}\right)_{j_{f}}^{i_{f}} U_{ \pm, b_{f}}^{\dagger j_{f}} \psi_{f, \beta_{f}}^{b_{f}}\left(q_{f}\right)\right. \\
& \times Q_{a_{3}}^{+} U_{ \pm, i_{3}}^{a_{3}}\left(\theta^{-1}\left(w_{ \pm}-\theta\right) \theta^{-1}\right)_{j_{3}}^{i_{3}} U_{ \pm, b_{3}}^{\dagger j_{3}} Q^{b_{3}} .
\end{aligned}
$$

It is evident that the integration over $z$ leads to the energy-momentum conservation delta-function, while the integration over color orientation provides the specific structure of the interaction terms. Also, each light quark leg is accompanied by the form-factor $F=F(k \rho)$, which is localized at the region $k \rho \leq 1$, as expected.

Details of $Q^{+}\left[\theta^{-1}\left(w_{ \pm}-\theta\right) \theta^{-1}\right] Q$.

We take initial and final positions of a heavy quarks at $x=\left(\vec{x}, t_{1}\right)$ and $x^{\prime}=\left(\vec{x}, t_{2}\right)$ and have

$$
<\vec{x}, t_{2}\left|\left[\theta^{-1}\left(w_{ \pm}-\theta\right) \theta^{-1}\right]\right| \vec{x}, t_{1}>=\frac{d}{d t_{1}} \frac{d}{d t_{2}} \Theta\left(t_{2}-t_{1}\right)\left[P \exp \left(i \int_{t_{1}}^{t_{2}} A_{ \pm, 4} d x_{4}\right)-1\right] .
$$

The next step is to calculate P-exponent taking singular gauge for the (anti)instanton at the position $z$.

Then, representing $Q(\vec{x}, t)=\int \frac{d \omega d^{3} \vec{p}}{(2 \pi)^{4}} Q(\vec{p}, \omega) \exp [i(\vec{p} \vec{x}+\omega t)]$ we find

$$
\begin{aligned}
& \int d t_{1} d t_{2} d^{3} x Q^{+}\left(\vec{x}, t_{2}\right)<\vec{x}, t_{2}\left|\left[\theta^{-1}\left(w_{ \pm}-\theta\right) \theta^{-1}\right]\right| \vec{x}, t_{1}>Q\left(\vec{x}, t_{1}\right) \\
& =\int \frac{d \omega_{1} d^{3} p_{1}}{(2 \pi)^{4}} \frac{d \omega_{2} d^{3} p_{2}}{(2 \pi)^{4}} e^{i \vec{p} \vec{z}} Q^{+}\left(\vec{p}_{2}, \omega_{2}\right)\left(J_{0}(p \rho) \mp \vec{\tau} \vec{m} J_{1}(p \rho)\right) Q\left(\vec{p}_{1}, \omega_{1}\right) .
\end{aligned}
$$

Here $\vec{p}=\vec{p}_{1}-\vec{p}_{2}, \vec{m}=\vec{p} / p$ and

$$
\begin{aligned}
& J_{0}(y)=-8 \pi \rho^{3} i_{0}(y), i_{0}(y)=\int_{0}^{\infty} d z \sin [z y] / z y\left(z \cos \left[\pi z / 2\left(z^{2}+1\right)^{0.5}\right]\right)^{2}, i(0)=0.552, \\
& J_{1}(y)=4 \pi \rho^{3} i_{1}(y), i_{1}(y)=\int_{0}^{\infty} d z z^{2}\left(\cos [z y] / z y-\sin [z y] / z^{2} y^{2}\right) \sin \left[\pi z /\left(z^{2}+1\right)^{0.5}\right]
\end{aligned}
$$

These form-factors are localized in the region $y=p \rho \leq 1$, as expected. 


\section{Heavy quark light mesons interaction term}

Equation (4.2) has an essential part containing the co-product of colorless heavy-quark factor and the colorless light-quark one:

$$
\begin{aligned}
& S\left[\psi, \psi^{+}, Q^{+} Q\right]=i \lambda \int d^{4} x \exp (-i p x) \frac{d^{3} p_{1} d \omega_{1}}{(2 \pi)^{4}} \frac{d^{3} p_{2} d \omega_{2}}{(2 \pi)^{4}} \frac{16 \pi \rho^{3}}{N_{c}} i_{0}(p \rho) Q^{+}\left(\vec{p}_{2}, \omega_{2}\right) Q\left(\vec{p}_{1}, \omega_{1}\right) \\
& \frac{1}{8\left(N_{c}^{2}-1\right)}\left[\left(1-\frac{1}{2 N_{c}}\right)\left(q^{+}(x) q(x) q^{+}(x) q(x)+q^{+} \gamma_{5} q q^{+} \gamma_{5} q-q^{+} \vec{\tau} q q^{+} \vec{\tau} q-q^{+} \gamma_{5} \vec{\tau} q q^{+} \gamma_{5} \vec{\tau} q\right)\right. \\
& \left.-\frac{1}{8 N_{c}}\left(q^{+} \sigma_{\mu \nu} q q^{+} \sigma_{\mu \nu} q+q^{+} \gamma_{5} \sigma_{\mu \nu} q q^{+} \gamma_{5} \sigma_{\mu \nu} q-q^{+} \sigma_{\mu \nu} \vec{\tau} q q^{+} \sigma_{\mu \nu} \vec{\tau} q-q^{+} \gamma_{5} \sigma_{\mu \nu} \vec{\tau} q q^{+} \gamma_{5} \sigma_{\mu \nu} \vec{\tau} q\right)\right],
\end{aligned}
$$

where $q(x)=2 \pi \rho F(i \partial) \psi(x)$ and $q^{+}(x)=2 \pi \rho F(i \partial) \psi^{+}(x)$.

The application of the standard bosonization procedure to the light quarks and the calculation of the path integrals over $\lambda$ and meson fields in the saddle point approximation lead to the vacuum equations in the leading order (LO) on $1 / N_{c}$ expansion. It is natural that these mesons $(\sigma, \vec{\phi}, \ldots)$ have properties corresponding to light quarks bilinears $\left(q^{+} q, q^{+} \gamma_{5} \vec{\tau} q, \ldots\right)$.

So, the vacuum equations in the $\mathrm{LO}$ are written as

$$
\operatorname{Tr} \frac{M(p)}{\hat{p}+i(m+M(p))}=N=\sigma_{0}^{2} V, M(p)=M F^{2}(p), M^{2}=(2 \pi \rho)^{4} \lambda \frac{2 N_{c}-1}{2 N_{c}\left(N_{c}^{2}-1\right)} \sigma_{0}^{2} .
$$

They fix the coupling $\lambda$ and saddle-point $\sigma_{0}$ and accordingly the dynamical quark mass $M(p)=$ $M F^{2}(p)$.

Now the total scalar meson field is $\sigma=\sigma_{0}+\sigma^{\prime}$, where $\sigma^{\prime}$ is a quantum fluctuation. Other mesons are presented only by their quantum fluctuations.

At the saddle points we have the effective action for the mesons and colorless heavy quark $Q^{+} Q$ bilinear as

$$
\begin{aligned}
& S\left[\sigma^{\prime}, \vec{\phi}^{\prime}, \eta^{\prime}, \vec{\sigma}^{\prime}, Q^{+} Q\right]=-\operatorname{Tr} \ln \frac{\hat{p}+i(m+M(p))}{\hat{p}+i m}+N / 2
\end{aligned}
$$

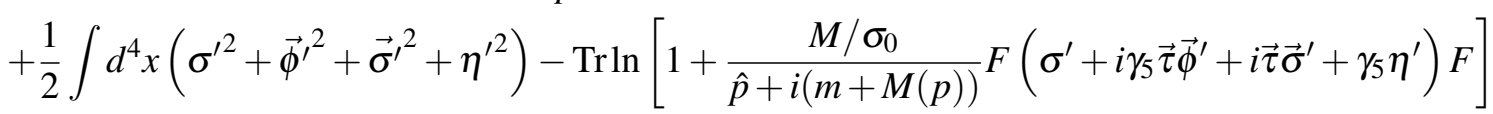

$$
\begin{aligned}
& -\operatorname{Tr} \frac{1}{\hat{p}+i(m+M(p))+\frac{M}{\sigma_{0}} F\left(\sigma^{\prime}+i \gamma_{5} \vec{\tau} \vec{\phi}^{\prime}+i \vec{\tau} \vec{\sigma}^{\prime}+\gamma_{5} \eta^{\prime}\right) F}\left(\frac{i}{2} \int e^{-i p x} \frac{d^{3} p_{1} d \omega_{1}}{(2 \pi)^{4}} \frac{d^{3} p_{2} d \omega_{2}}{(2 \pi)^{4}} \frac{2}{N_{c}} J_{0}(p \rho) Q^{+} Q\right) .
\end{aligned}
$$

The second line describe mesons and their interactions, while the third one explains the renormalization of the heavy quark mass and heavy-light quark meson interactions terms.

The renormalization of heavy quark mass (neglecting current light quark mass $\mathrm{m}$ ) is given by

$$
\begin{aligned}
& \Delta_{Q}=\operatorname{Tr} \frac{1}{\hat{p}+i M(p)}\left(\frac{i}{2} \int e^{-i p x} \frac{d^{3} p_{1} d \omega_{1}}{(2 \pi)^{4}} \frac{d^{3} p_{2} d \omega_{2}}{(2 \pi)^{4}} \frac{2}{N_{c}} J_{0}(p \rho) Q^{+} Q\right) \\
& =8 \int \frac{d^{4} p}{(2 \pi)^{4}} \frac{M(p)}{p^{2}+M^{2}(p)}\left(\int \frac{d^{3} p_{1} d \omega_{1}}{(2 \pi)^{4}} J_{0}(0) Q^{+}\left(\vec{p}_{1}, \omega_{1}\right) Q\left(\vec{p}_{1}, \omega_{1}\right)\right) .
\end{aligned}
$$

Taking into account Eq. (5.2), we have

$$
\Delta_{Q}=\frac{2}{N_{c}} \frac{N}{V} J_{0}(0)\left(\int \frac{d^{3} p_{1} d \omega_{1}}{(2 \pi)^{4}} Q^{+}\left(\vec{p}_{1}, \omega_{1}\right) Q\left(\vec{p}_{1}, \omega_{1}\right)\right) .
$$


So, the instanton media contribution to the heavy quark mass is

$$
\Delta M=-2 J_{0}(0) / N_{c} R^{4}=16 \pi i_{0}(0)\left(\rho^{4} / R^{4}\right) \rho^{-1} / N_{c},
$$

in complete coincidence with [7] as we expected.

If we take the following values: $\rho=0.35 \mathrm{fm}, R=0.856 \mathrm{fm}$ (1.3), we obtain $M=570 \mathrm{MeV}$ and $\Delta M=148 \mathrm{MeV}$. These factors define the coupling between heavy and light quarks in Eq. (4.2) and certainly between heavy quarks and light mesons in Eq. (5.3).

\section{Conclusion}

The instanton vacuum generates a specific interaction not only between light quarks but also between light and heavy quarks. All of the features of these interaction terms are completely defined by the instanton media parameters $\rho$ and $R$. It is natural to apply this approach to heavy quark and heavy-light quark systems and predict the properties of these systems. This is a program of our future research.

\section{Acknowledgments}

This work was done in a close collaboration with Hyun-Chul Kim and his group from Inha University (Korea). I am thankful to him for many detailed discussions. I acknowledge the support from the grant F2-60 of Uzbekistan State Committee for Science and Technology.

\section{References}

[1] D. Diakonov, Prog. Part. Nucl. Phys. 51, 173 (2003).

[2] T. Schafer and E. V. Shuryak, Rev. Mod. Phys. 70 (1998) 323.

[3] M. C. Chu, J. M. Grandy, S. Huang and J. W. Negele, Phys. Rev. D 49 (1994) 6039;

J. W. Negele, Nucl. Phys. Proc. Suppl. 73 (1999) 92;

T. DeGrand, Phys. Rev. D 64 (2001) 094508;

P. Faccioli and T. A. DeGrand, Phys. Rev. Lett. 91 (2003) 182001;

P. O. Bowman, U. M. Heller, D. B. Leinweber, A. G. Williams and J. b. Zhang, Nucl. Phys. Proc. Suppl. 128 (2004) 23.

[4] M. Cristoforetti, P. Faccioli, M. C. Traini and J. W. Negele, Phys. Rev. D 75 (2007) 034008.

[5] H. Leutwyler, Czech. J. Phys. 52 (2002) B9.

[6] K. Goeke, M. M. Musakhanov and M. Siddikov, Phys. Rev. D 76 (2007) 076007.

[7] D. Diakonov, V. Y. Petrov and P. V. Pobylitsa, Phys. Lett. B 226 (1989) 372.

[8] S. Chernyshev, M. A. Nowak and I. Zahed, Phys. Rev. D 53 (1996) 5176.

[9] D. Diakonov, V. Polyakov and C. Weiss, Nucl. Phys. B 461 (1996) 539.

[10] P. V. Pobylitsa, Phys. Lett. B 226 (1989) 387. 
[11] M. M. Musakhanov and F. C. Khanna, Phys. Lett. B 395 (1997) 298;

E. D. Salvo and M. M. Musakhanov, Eur. Phys. J. C 5(1998)501;

M. Musakhanov, Eur. Phys. J. C 9 (1999) 235 ;

M. Musakhanov, Nucl. Phys. A 699(2002) 340;

M. M. Musakhanov and H. C. Kim, Phys. Lett. B 572 (2003) 181;

H. C. Kim, M. Musakhanov and M. Siddikov, Phys. Lett. B 608 (2005) 95;

H. C. Kim, M. M. Musakhanov and M. Siddikov, Phys. Lett. B 633 (2006) 701;

K. Goeke, H. C. Kim, M. M. Musakhanov, M. Siddikov, Phys. Rev. D 76 (2007) 116007;

K. Goeke, M. Musakhanov, M. Siddikov, Phys. Rev. D 81 (2010) 054029. 